\title{
Decentralized reactive power dispatch for a time-varying multi-TSO system
}

\author{
Yannick Phulpin \\ Supelec, France \\ yannick.phulpin@supelec.fr miroslav@ece.gatech.edu \\ Miroslav Begovic \\ Georgia Tech, USA
}

yannick.phulpin@supelecti miroslav@ece.gatech.edu 1

\author{
Marc Petit \\ Supelec, France \\ marc.petit@supelec.fr \\ Damien Ernst \\ University of Liège, Belgium \\ ernst@montefiore.ulg.ac.be
}

\begin{abstract}
This paper addresses the problem of reactive power dispatch in a power system partitioned into several areas controlled by different transmission system operators. Previous research has shown that nearly optimal performance could be achieved in a time-invariant system by using a specific iterative decentralized control scheme with no information exchange. At each iteration of this scheme, every transmission system operator concurrently schedules its own control settings for the next iteration while representing the neighboring areas with external network equivalents. This paper focuses on some parameter tracking techniques to extend the range of application of the decentralized control scheme to time-varying systems, where the time-varying nature of the system is modeled as a succession of steady-state operating conditions with variation of the load demand. Those new techniques are evaluated in the context of IEEE 118 bus system partitioned into three control areas.
\end{abstract}

\section{Introduction}

Secure operation of power systems requires appropriate coordination of the control actions, which has motivated the development of sophisticated control schemes [1]. Hence, most transmission system operators (TSOs) have specifically implemented voltage control in their own control area. Some examples are reported in [2]-[4], which detail some practices within Northern America and Europe.

While dynamics issues have been locally addressed with automatic voltage regulation [5], some recent incidents raise the problem of the steady-state control of large-scale systems [6]. More specifically, a major issue is to coordinate the operation scheduling of the different TSOs so as to achieve high performance in the interconnected networks [7]. While many authors deal with reactive power dispatch issues for single-TSO systems (e.g. [8], [9]), multi-TSO system operation has lately been receiving increasing attention. Actually, several authors have emphasized the potential benefits of a coordinating control center [10], [11] and decentralized control schemes [12]-[14] to address multiTSO operation issues.

In [14], a decentralized scheme is proposed, where the different TSOs concurrently schedule reactive power dispatch within their own control area while representing the neighboring areas with external network equivalents, whose parameters are fitted based on local measurements only. In the context of a time-invariant system, it is demonstrated that this scheme achieves nearly optimal performance. However, its performance is affected when the power system is time varying (non constant load, sudden loss of transmission element, etc).

To improve the decentralized scheme in this context, this paper proposes a new procedure to compute the external network equivalents' parameters. Unlike in [14], which considers time-invariant power systems only, we consider a succession of steady-state conditions and iteratively optimize the control settings for those situations. In this context, we propose to rely on some adaptive tracking strategies to compute the external network equivalents' parameters. As outlined in [15], those tracking strategies have already been widely used to design adaptive control schemes for power systems. In particular, they have led to the design of efficient control strategies for damping interarea oscillations [16], [17]. Three particular types of adaptive fitting techniques will be proposed in this paper. They will be evaluated and compared on the IEEE 118 bus system, where three control areas have been defined.

The paper is organized as follows. In Section 2, the reactive power dispatch problem is formalized and the benchmark power system is described. In Section 3, a clear description is given of the decentralized control scheme proposed in [14]. Afterwards, some methods for fitting parameters of the equivalents are proposed 
to extend the range of applications of the decentralized control scheme to time-varying system models. In Section 4, an empirical evaluation of those methods is carried out. Finally, Section 5 concludes and discusses some future research directions.

\section{Problem formulation}

In this section, the multi-TSO reactive power dispatch problem is described. Further, the benchmark power system that will serve to assess the potential performance of the decentralized control scheme is presented.

\subsection{Centralized problem}

As introduced in [9], some TSOs optimize the voltage settings across their network while considering a stationary load demand and generation dispatch. This practice is not rare and many TSOs even use more heuristic rules for reactive power dispatch [2].

However, we consider in this paper that every TSO computes at instant $k-1$ the voltage settings that will be applied at instant $k$ to the generators and compensators located in its control area. In a single-TSO system, the optimal power flow problem (OPF) [18], [19] faced by the TSO at instant $k-1$ can be written as follows

$$
\min _{\mathbf{u}, \mathbf{x}} C^{k}(\mathbf{u}, \mathbf{x})
$$

under the inequality and equality constraints

$$
\begin{aligned}
\mathbf{f}^{k}(\mathbf{u}, \mathbf{x}) & \leq 0 \\
\mathbf{g}^{k}(\mathbf{u}, \mathbf{x}) & =0
\end{aligned}
$$

where $\mathbf{u}$ and $\mathbf{x}$ are vectors of control variables and state variables, respectively, $C^{k}(\mathbf{u}, \mathbf{x})$ is the objective function and $f^{k}(\mathbf{u}, \mathbf{x})$ and $g^{k}(\mathbf{u}, \mathbf{x})$ represent the constraint functions. The superscript $k$ denotes the fact that those functions depend on the scheduled active power generation dispatch, load demand and network topology at time $k$. In the context of reactive power dispatch, the practical meaning of the different terms used to formalize the optimization problem is detailed hereafter.

The control variable vector $\mathbf{u}$ usually represents the tap ratios of the transformers and the voltage settings of generators and compensation devices [20]. The state variable vector $\mathbf{x}$ represents the bus voltage angles and magnitudes.
As the choice of the objective function is particularly strategic, many works focus on its formulation. While implicit objectives are mainly concerned with the operational costs and the level of security, those can be explicitly reflected through different criteria, which are mainly reported in [8]. More specifically, common practices are to focus on active power losses $A P L^{k}$ or on reactive power support $R P S^{k}$, for which a formulation is proposed as follows

$$
\operatorname{RPS}^{k}(\mathbf{u}, \mathbf{x})=\sum_{j=1}^{N_{G}} Q_{j}(k)^{2}
$$

$Q_{j}(k)$ represents here the reactive power injection of generator $j$ at instant $k, N_{G}$ the number of generators. Some TSOs may also use a weighted sum of active power losses and of reactive power support. However, this kind of weighted objective function will not be studied in this paper.

Finally, Inequality (2) represents the constraints on the power system (e.g. bus voltage limits, transmission line current limits, minimum and maximum power production, etc), while the Equality (3) has mainly been introduced to represent power flow equations and the distributed loss compensation (distributed slackbus).

\subsection{Multi-TSO problem}

As noted in [14], in the context of multi-TSO reactive power dispatch, a new constraint must be added to the set of equality constraints (3) to set active power export at its scheduled level at time $k$.

In the following, we will consider a power system with NbTSO TSOs referred to as the subscripts $1,2, \ldots, N b T S O$. We will also suppose that the constraint functions $f^{k}(\mathbf{u}, \mathbf{x})$ and $g^{k}(\mathbf{u}, \mathbf{x})$ can be decomposed into NbTSO constraint functions $f_{1}^{k}(\mathbf{u}, \mathbf{x})$, $f_{2}^{k}(\mathbf{u}, \mathbf{x}), \ldots, f_{N b T S O}^{k}(\mathbf{u}, \mathbf{x})$ and $g_{1}^{k}(\mathbf{u}, \mathbf{x}), g_{2}^{k}(\mathbf{u}, \mathbf{x}), \ldots$, $g_{\text {NbTSO }}^{k}(\mathbf{u}, \mathbf{x})$, respectively, where each constraint function $f_{i}^{k}(\mathbf{u}, \mathbf{x})$ or $g_{i}^{k}(\mathbf{u}, \mathbf{x})$ corresponds to the constraints associated with $T S O_{i}$.

Since the TSOs mostly focus on their own area, they usually have different objective functions. However, to compare the results obtained in a multiTSO context with those that would be obtained if the TSOs had merged into a single entity, we will assume in this paper that every TSO has the same type of objective. This means that the cost function $C^{k}(\mathbf{u}, \mathbf{x})$ can be expressed as the sum of NbTSO functions $C_{1}^{k}(\mathbf{u}, \mathbf{x}), C_{2}^{k}(\mathbf{u}, \mathbf{x}), \ldots, C_{N b T S O}^{k}(\mathbf{u}, \mathbf{x})$ where each function $C_{i}^{k}(\mathbf{u}, \mathbf{x})$ has the same nature (i.e. $A P L^{k}$ or $\left.R P S^{k}\right)$. 


\subsection{Benchmark system}

The benchmark power system used herewith is the IEEE 118 bus system (presented in [21], for example), which has been partitioned into three areas referred to as 1,2 , and 3. This system is shown in Figure 1.

We model the time-varying load demand by a discrete-time variation of the load demand, and consider that the "real" time interval between two successive discrete instants $k-1$ and $k$ is 30 minutes. At each instant $k$, the active power demand $P_{D_{j}}(k)$ and the corresponding reactive power demand $Q_{D_{j}}(k)$ are obtained by multiplying their respective initial values by a load factor $r(k)$. They can thus be expressed as follows

$$
\begin{gathered}
P_{D_{j}}(k)=P_{D_{j}}(0) \times r(k) \\
Q_{D_{j}}(k)=Q_{D_{j}}(0) \times r(k)
\end{gathered}
$$

where $P_{D_{j}}(0)$ and $Q_{D_{j}}(0)$ represent the active and reactive power demand as defined in [21], respectively.

For a convenient and realistic modeling of the load demand variations in the IEEE 118 bus system, the load factor $r(k)$ is associated with real observations on the French power system during the period January 1st-31st, 2008. More precisely, we have averaged the French power demand over this period and computed the ratios $r(k)$ between the demand at the different instants $k$ and this average. We note that, in a real system, $r(k)$ could be accurately predicted at instant $k-1$ using short-term load forecasting methods. We will assume from now on that no prediction error occurs in this case.

We assume that the active power injections homothetically grow with the load factor. However, a decentralized slack bus is used in our simulations, which may slightly change the generation pattern depending on active power losses. All the control variables in $\mathbf{u}$ are continuous, and represent the generators' and non discrete compensation devices' voltage settings.

The period January 1st-26th 2008 will serve as a "training period" for the adaptive control scheme, and simulation results will only focus on the period January 27th-31st 2008. To consider fast changes in the system configuration, a line outage is introduced for the branch between bus 19 and 20 on January 28th at noon. The line is reconnected on January 29th at noon.

\section{Decentralized control scheme}

As mentioned in [13], a simple approach to address the problem of multi-TSO operation is to propose a decentralized coordination scheme where every TSO

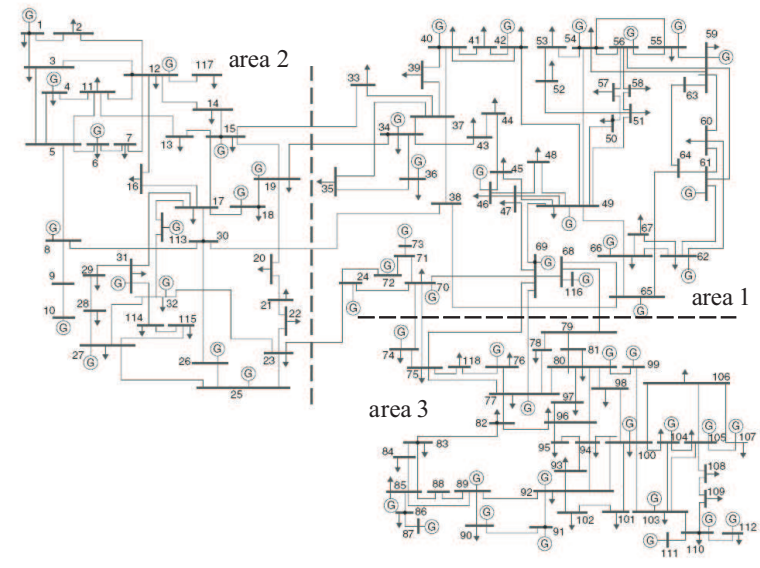

Figure 1. IEEE 118 bus system with three TSOs.

maintains its prerogatives (objective function, control actions, load forecasting, etc) and optimizes its own control area with respect to a specific procedure. Such a procedure is detailed hereafter, in the context of reactive power dispatch.

\subsection{Decentralized algorithm}

The main features of the decentralized control scheme proposed in [14], and adapted here to timevarying systems, are sketched on Figure 2. The scheme is iterative in nature, and every TSO concurrently solves at time $k-1$ the scheduling problem corresponding to its own area at time $k$, and then, applies its control actions on the system at time $k$.

The coordination relies on the fact that every TSO is recommended to model the external system with a set of parametric equality constraints $h_{i}^{k}\left(\mathbf{u}_{i}, \mathbf{x}_{i}, \mathbf{z}_{i}^{*}(k)\right)$, which correspond to a simple external network model (ENM) whose parameters are denoted by $\mathbf{z}_{i}^{*}(k)$, and to solve the optimization problem corresponding to its control area in a greedy way. The scheme is obviously simple since, among others, it requires no need for communication between the different TSOs or for a centralized authority to coordinate their actions.

While different types of ENM could be advocated, we propose to focus in this paper on the equivalent that performs best in time-invariant conditions. Hence, we use the constant PQ equivalent, whose higher performance is demonstrated in [14]. In such a context, $\mathbf{z}_{i}^{*}(k)$ is a $2 \times N b I_{i}$ component vector, where $N b I_{i}$ represents the number of interconnection lines between $T S O_{i}$ and the other TSOs. It gathers all the values of active power and reactive power parameters of the PQ equivalents used by $T S O_{i}$ to represent the external system. 


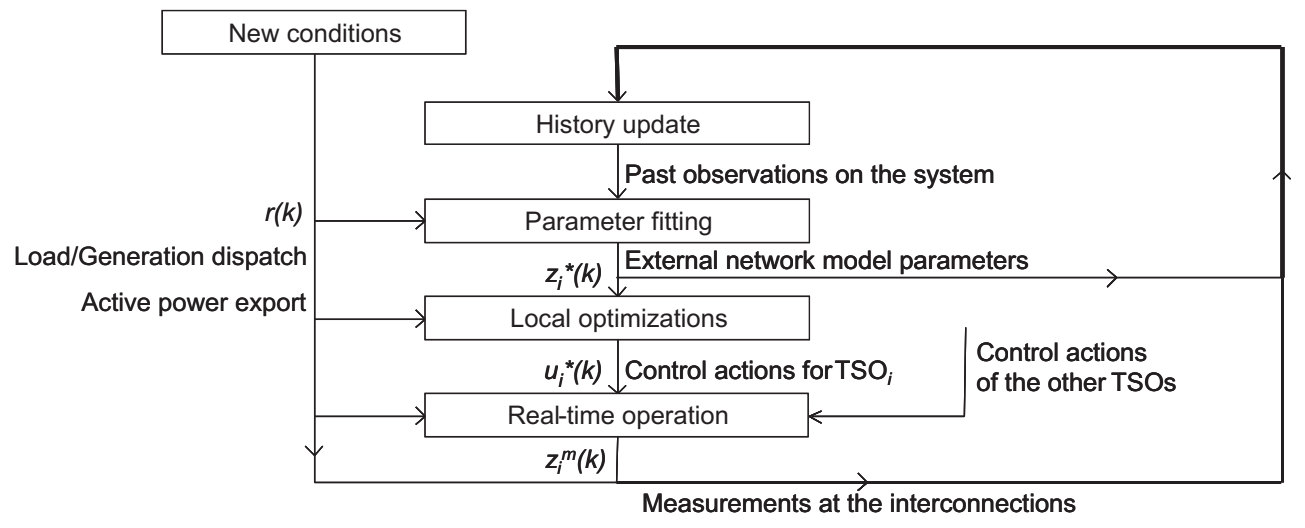

Figure 2. The role of $\mathrm{TSO}_{i}$ in the decentralized optimization scheme.

At instant $k-1$, every $T S O_{i}$ thus solves the following optimization problem

$$
\min _{\mathbf{u}_{i}, \mathbf{x}_{i}} C_{i}^{k}\left(\mathbf{u}_{i}, \mathbf{x}_{i}\right)
$$

under the inequality and equality constraints

$$
\begin{aligned}
\mathbf{f}_{i}^{k}\left(\mathbf{u}_{i}, \mathbf{x}_{i}\right) & \leq 0 \\
\mathbf{g}_{i}^{k}\left(\mathbf{u}_{i}, \mathbf{x}_{i}\right) & =0 \\
\mathbf{h}_{i}^{k}\left(\mathbf{u}_{i}, \mathbf{x}_{i}, \mathbf{z}_{i}^{*}(k)\right) & =0
\end{aligned}
$$

The solutions $\mathbf{u}_{1}^{*}(k), \mathbf{u}_{2}^{*}(k), \ldots, \mathbf{u}_{N b T S O}^{*}(k)$ are appended onto $\mathbf{u}^{*}(k)$, which is applied to the interconnected system at instant $k$.

It may happen that the actions $\mathbf{u}_{1}^{*}(k), \mathbf{u}_{2}^{*}(k), \ldots$, $\mathbf{u}_{N b T S O}^{*}(k)$ correspond to a state that does not satisfy ${ }^{1}$ constraints (2) and (3). Usually, as shown in [14], the constraints, if violated, are only slightly "passed over," and, in practice, some dedicated controllers may accordingly change the operation conditions of the system to make sure that the constraints are satisfied. In our simulation environment, we will consider that such controllers exist, and that their combined action is equivalent to choosing, instead of $\mathbf{u}^{*}(k)$, a vector of control variables $\mathbf{u}^{m}(k)$ solution of

$$
\min _{\mathbf{u}, \mathbf{x}}\left\|\mathbf{u}^{*}(k)-\mathbf{u}\right\|
$$

under Inequality (2) and Equality (3). Active power and reactive power flows at each interconnection, represented by $\mathbf{z}_{i}^{m}(k)$, are then measured by each TSO, and the history of measurements at the interconnections is updated.

We will suppose in this paper that every TSO proceeds fairly, according to the recommended procedure, and uses the same type of equivalent and procedure to compute its parameters $\mathbf{z}_{i}^{*}(k)$.

1. This may be checked in a simulation environment by running a load flow.

\subsection{Adaptive parameter fitting}

As two key elements of the decentralized control scheme, there are the equivalents used by every TSO to represent its neighboring areas and the procedure to fit the parameters of those equivalents 'at best' so that the final allocation leads to nearly optimal performance.

As represented in Figure 2, several types of inputs may be considered in the design of parameter tracking procedure. Those are, for example, the past observations of the power system, load predictions, and past values of the equivalents' parameters. In this paper, for computing the parameters of a PQ equivalent related to a particular interconnection, we will only consider as input the past measurements of active and reactive power at this interconnection. This strategy is also used in [14]. The parameter tracking issue is tackled by using three different least squares-based fitting approaches, which are detailed hereafter.

3.2.1. Exponential recursive least squares approach. The exponential recursive least squares (ERLS) strategy computes $\mathbf{z}_{i}^{*}(k)$ by solving the following miminization problem

$$
\min _{\mathbf{z}} \sum_{j=0}^{k-1} \beta^{1+j-k} \times\left\|\mathbf{z}^{m}(j)-\mathbf{z}\right\|^{2}
$$

where $\beta$ is a memory factor such that $\beta \in[0,1]$, and $\mathbf{z}_{i}^{m}(j)$ the parameters' on-site measurement at instant $j$. As the choice of the memory factor $\beta$ affects the value of $\mathbf{z}_{i}^{*}(k)$, this choice may be subject to some tuning. This approach was used in [14] to fit the parameters of the equivalents, and was shown to be successful for time-invariant power system models. 
3.2.2. Environment-dependent exponential recursive least squares approach. While the ERLS approach emphasizes the importance of recent measurements, those measurements may correspond to a power system state that is particularly different from the scheduled one. A solution could be to use an environment-dependent exponential recursive least squares approach (ED-ERLS), where the memory factor $\gamma(k, j)$ would weight the measurements at instant $j$ according to the similarity between the power system state at time $j$ and the one scheduled for time $k$. For the sake of simplicity, we consider in this paper that the state at instant $j$ is similar to the state at instant $k$ if the associated load factor $r(j)$ is close to $r(k)$.

The term $\gamma(k, j)$ can thus be typically written as follows

$$
\gamma(k, j)=N_{r(k)}^{\sigma}(r(j))
$$

where $N_{r(k)}^{\sigma}(\cdot)$ is a Gaussian function with mean $r(k)$ and variance $\sigma$.

In this context, $\mathbf{z}_{i}^{*}(k)$ is the solution of the following problem

$$
\min _{\mathbf{z}} \sum_{j=0}^{k-1}[\gamma(k, j)]^{1+j-k} \times\left\|\mathbf{z}^{m}(j)-\mathbf{z}\right\|^{2}
$$

3.2.3. Adaptive forgetting factor approach. The previous approaches do not allow a fast tracking of specific changes in the system configuration (e.g. change in the system topology), which could significantly affect the steady-state values of the parameters at the interconnections. Therefore, we propose an adaptive forgetting factor (AFF) approach, which is introduced in [22] for tracking non-linear systems in both slow and fast time-varying environments. The rationale of this method is to use, as weighting factor, the product of two terms $\psi(k) \times \gamma(k, j)$, where $\gamma(k, j)$ is defined by Eqn (13), and where $\psi(k) \in] 0,1[$ is close to 1 , when there is no fast change in the system between instants $k-1$ and $k$, and close to 0 otherwise.

More specifically, $\psi(k)$ depends on the prediction error observed at time $k-1, \epsilon_{i}(k-1)=$ $\left\|\mathbf{z}_{i}^{m}(k-1)-\mathbf{z}_{i}^{*}(k-1)\right\|$, in the following way

$$
\psi(k)=\exp \left(-\tau \times \epsilon_{i}(k-1)\right)
$$

where $\tau$ is a forgetting factor.

Therefore, $\mathbf{z}_{i}^{*}(k)$ is the solution of the following problem

$$
\min _{\mathbf{z}} \sum_{j=0}^{k-1}[\psi(k) \times \gamma(k, j)]^{1+j-k} \times\left\|\mathbf{z}^{m}(j)-\mathbf{z}\right\|^{2}
$$

\section{Simulation results}

The adaptive parameter tracking procedures are evaluated on the IEEE 118 bus system with three TSOs introduced in Section 2.3. Since a "training period" is necessary to initialize the history of measurements and control variables, only results corresponding to the period January 27th-31st, 2008 are presented.

\subsection{Evaluation index}

The decentralized optimization scheme is said to perform well, if the control setting $\mathbf{u}^{m}(k)$ that is applied at every instant $k$ is close to the optimal control settings $\mathbf{u}^{*}(k)$ that would be computed if all TSOs had merged into a single entity. In this paper, the distance between $\mathbf{u}^{m}(k)$ and $\mathbf{u}^{*}(k)$ is computed as the difference between $C\left(\mathbf{u}^{m}(k)\right)$ and $C\left(\mathbf{u}^{*}(k)\right)$. More specifically, the suboptimality measure at instant $k$ is assessed as follows

$$
S O(k)=\frac{C\left(\mathbf{u}^{m}(k)\right)-C\left(\mathbf{u}^{*}(k)\right)}{\underset{k}{\operatorname{mean} C\left(\mathbf{u}^{*}(k)\right)}}
$$

Although it is informative to study the evolution of $S O(k)$ with respect to $k$, we propose, for a convenient analysis of the performance of the control scheme, to focus on the average suboptimality measure $A S O$ over the period January 27th-31st, 2008.

The $A S O$ obtained with the different parameter fitting techniques will be compared with the average suboptimality measure over $k$ that would be obtained if the control scheme proposed in [14] were to iterate an infinite number of times for every operating state corresponding to instant $k$. When minimizing active power losses, this average suboptimality is equal to $0.27 \%$, and it amounts $9.27 \%$ for the minimization of reactive power support.

\subsection{Performance with ERLS-algorithm}

Figure 3 depicts the average suboptimality measure obtained with an ERLS-algorithm when minimizing active power losses. One can observe that the memory factor $\beta$ has a small influence on the $A S O$ when the objective function is the minimization of active power losses. In this case, the performance of the decentralized control scheme is particularly close to the one that would be obtained by iterating the scheme an infinite number of times for every instant $k$. In particular, the average suboptimality measure amounts $0.29 \%$ with $\beta=0.1$.

As the average suboptimality measure is much higher with the other objective function under consideration, the remainder of this section will mainly 


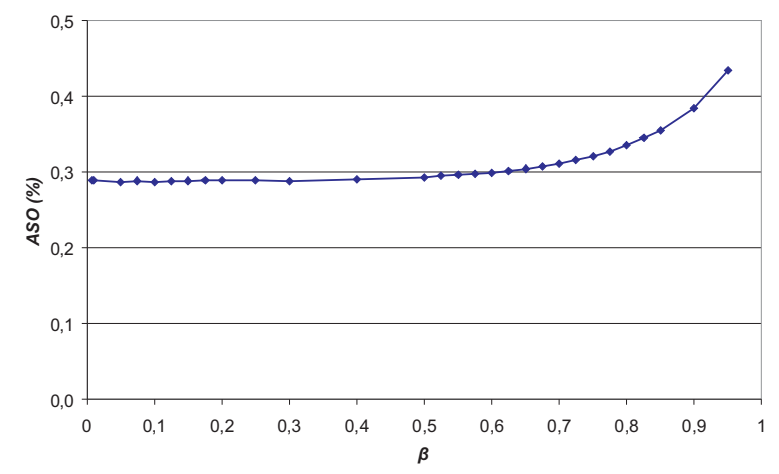

Figure 3. Average suboptimality index as a function of $\beta$ for the minimization of active power losses through the decentralized control scheme with an ERLS fitting algorithm.

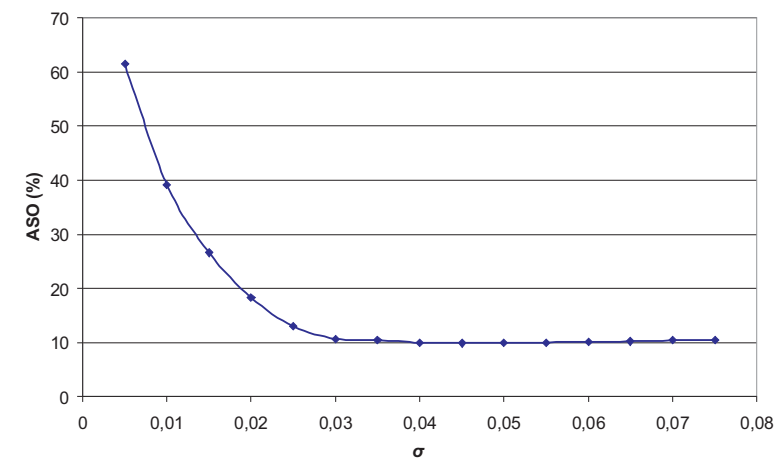

Figure 5. Average suboptimality index as a function of $\sigma$ for the minimization of reactive power support through the decentralized control scheme with an ED-ERLS fitting algorithm.

focus on the minimization of reactive power support. Figure 4 presents the average subotpimality index obtained with an ERLS-algorithm and the minimization of reactive power support. The lowest $A S O$ is reported for $\beta=0.575$. Even with such a memory factor, the $A S O$ is significantly higher than the one that would be obtained by iterating the scheme an infinite number of times for every instant $k$ ( $11.49 \%$ vs $9.27 \%)$.

\subsection{Performance with ED-ERLS-algorithm}

The performance of the decentralized control scheme with an ED-ERLS parameter fitting procedure, when the objective is to minimize reactive power support, is reported on Figure 5. The impact of the

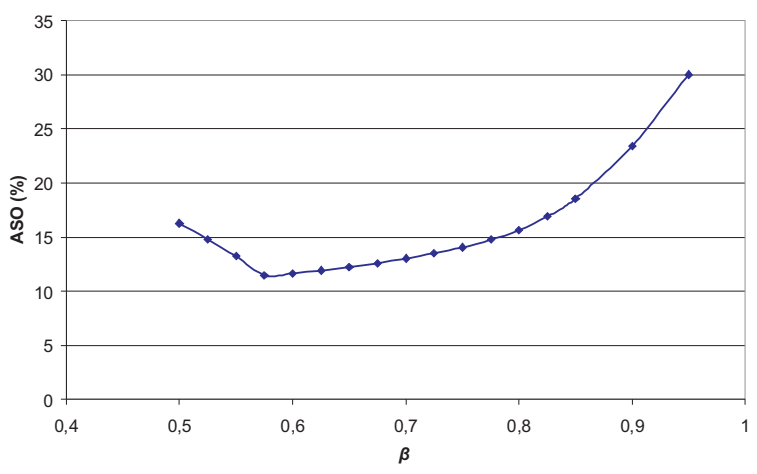

Figure 4. Average suboptimality index as a function of $\beta$ for the minimization of reactive power support through the decentralized control scheme with an ERLS fitting algorithm.

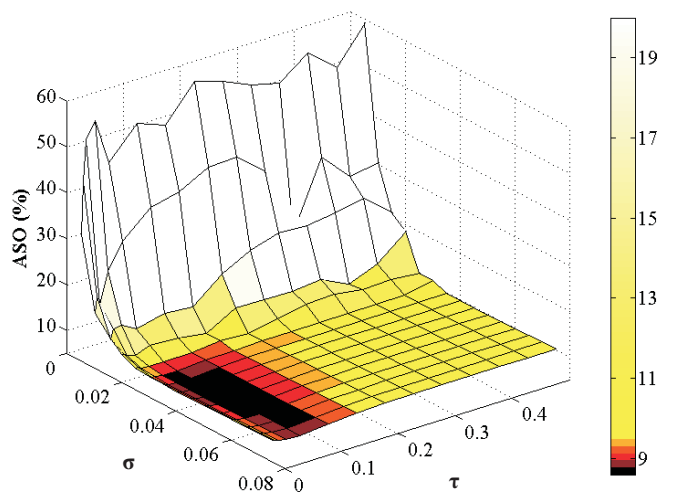

Figure 6. Average suboptimality index as a function of $\sigma$ and $\tau$ for the minimization of reactive power support through the decentralized control scheme with an AFF fitting algorithm.

similarity factor $\sigma$ is important, and one can observe that the best performance is reached with $\sigma \simeq 0.04$. In this case, the average suboptimality index, $A S O$, is around $9.43 \%$, which represents a considerable improvement over the performance observed with the ERLS algorithm.

\subsection{Performance with AFF-algorithm}

The performance of the decentralized control scheme with an AFF parameter fitting procedure when the objective is to minimize reactive power support is reported on Figure 6. This figure highlights the impact of the similarity factor $\sigma$ and of the deviation factor $\tau$ on the performance of the control scheme. 
More specifically, in this case, the best performance is reached with $\sigma=0.04$ and $\tau=0.025$. Those values of $\sigma$ and $\tau$ lead to an average suboptimality index $A S O$ equal to $8.59 \%$, which is even better than the average suboptimality that would be obtained by iterating the scheme an infinite number of times for every instant $k$. However, in real systems, $\sigma$ and $\tau$ would need to be fitted in real-time, and the performance of the decentralized control scheme would certainly be worse.

\section{Conclusion}

We have addressed in this paper the problem of decentralized optimization of multi-TSO power systems where the load demand evolves according to a discretetime load curve. Further development of a previously proposed decentralized control scheme (see [14]) has been presented. In this scheme, the TSOs assume an external network equivalent for their neighboring areas at the interconnections, and optimize at every iteration their control actions in a greedy way, i.e. without taking into consideration the impact that their actions may have on the other TSOs objectives. The range of application of this scheme has been extended to timevarying power systems by using parameter tracking techniques to better fit the parameters of the external network equivalents.

Although the control scheme does not require any explicit coordination and communication between the different TSOs, it achieves nearly optimal performance in the context of the minimization of active power losses, even with the original, and more simple, fitting techniques of [14]. Therefore, in this context, the need for sophisticated parameter tracking techniques is limited. On the other hand, we have shown that the simple adaptive parameter fitting technique of [14] does not lead to sufficient performance, when all TSOs aim to minimize reactive power support in their own area. In this context, the newly introduced parameter tracking methods lead to decentralized control schemes that achieve significantly better performance. In particular, the decentralized control scheme with an adaptive forgetting factor fitting procedure appears as an intriguing potential alternative to schedule reactive power dispatch in multi-TSO power systems. However, we believe that, prior to applying this decentralized control scheme to real systems, further investigation is still needed.

First, we have observed that the performance of the decentralized scheme depends on the tuning of its parameters (e.g. the parameters $\sigma$ and $\tau$ significantly influence the performance of the AFF technique). Systematic methods should thus be designed to optimize those parameters in real systems.

Second, up to now, the decentralized scheme has only been evaluated on the IEEE 118 bus system. It would be interesting to assess its performance within the framework of a large-scale system, such as the UCTE system for example.

Third, further research should also investigate the performance of the scheme when the TSOs have individual objectives of different types (e.g. some TSOs focus on active power losses while others on reactive power support), as it usually occurs in real systems. Such a study, among others, would require the definition of a quantitative index of the performance of multi-party ressource allocations.

\section{Acknowledgment}

Damien Ernst is a Research Associate of the Belgian FNRS, from which he acknowledges the financial support.

\section{References}

[1] Y. Rebours, D. Kirschen, M. Trotignon, and S. Rossignol, "A survey of frequency and voltage control ancillary services - part i: technical features," IEEE Transactions on Power Systems, vol. 22, pp. 350-357, February 2007.

[2] C. Taylor, "Reactive power today, best practices to prevent blackouts," IEEE Power and Energy Magazine, vol. 4, pp. 104-102, September 2006.

[3] C. Noe, G. Richerme., G. Blanchon, and F. Maury, "Le reglage automatique de la tension du reseau de transport d'edf," Revue Generale de l'Electricite, pp. 333-347, 1972.

[4] N. Janssens, "Tertiary and secondary voltage control for the Belgian HV system," in Proceedings of the IEE Colloquium on International Practices in Reactive Power Control, (London, UK), pp. 81-84, April 1993.

[5] T. Van Cutsem and C. Vournas, Voltage Stability of Electric Power Systems. Kluwer Academic Publishers, 1998.

[6] J. Bialek, "Are blackouts contagious?," IEE Power Engineer, vol. 17, pp. 10-13, December 2003.

[7] C. Taylor, "Improving grid behaviour," IEEE Spectrum, vol. 36, pp. 40-45, June 1999.

[8] R. Salgado and M. Irving, "Framework for the analysis of reactive power dispatch in energy pools," IEE Proc. on Generation, Transmission, Distribution, vol. 151, pp. 167-174, March 2004. 
[9] N. Martins, N. Macedo, L. Lima, and H. Pinto, "Control strategies for multiple static var compensators in long distance voltage supported transmission systems," IEEE Transactions on Power Systems, vol. 8, pp. 1107-1117, August 1993.

[10] J. Aguado and V. Quintana, "Inter-utilities powerexchange coordination: a market-oriented approach," IEEE Transactions on Power Systems, vol. 16, pp. 513519, August 2001.

[11] Y. Phulpin, M. Begovic, M. Petit, and D. Ernst, "On the fairness of centralized decision-making strategies for multi-tso power systems," in Proceedings of the PSCC 2008, (Glasgow, Scotland), July 2008.

[12] A. Marinakis, M. Glavic, and T. Van Cutsem, "Control of phase shifting transformers by multiple transmission system operators," in Proceedings of the Power Tech 2007, (Lausanne, Switzerland), July 2007.

[13] A. Conejo and J. Aguado, "Multi-area coordinated decentralized DC optimal power flow," IEEE Transactions on Power Systems, vol. 13, pp. 1272-1278, November 1998.

[14] Y. Phulpin, M. Begovic, M. Petit, J. Heyberger, and D. Ernst, "Evaluation of network equivalents for voltage optimization in multi-area power systems," Technical Report, pp. 1-44, available at: www.supelec.fr/deptenergie/Recherche/paper_TR.pdf, 2008.

[15] D. Pierre, "A perspective on adaptive control of power systems," IEEE Transactions on Power Systems, vol. 2, pp. 387-395, May 1987.
[16] R. Majumder, B. Chaudhuri, and B. Pal, "A probabilistic approach to model-based adaptive control for daming of interarea oscillations," IEEE Transactions on Power Systems, vol. 20, pp. 367-374, February 2005.

[17] S. Jain, F. Khorrami, and B. Fardanesh, "Adaptive nonlinear excitation control of power systems with unknown interconnections," IEEE Transactions on Control Systems Technology, vol. 2, pp. 436-446, December 1994.

[18] H. Dommel and W. Tinney, "Optimal power flow solutions," IEEE Transactions on Power Apparatus and Systems, vol. PAS-87, no. 10, pp. 1866-1876, 1968.

[19] F. Capitanescu, M. Glavic, D. Ernst, and L. Wehenkel, "Interior-point based algorithms for the solution of optimal power flow problems," Electric Power Systems Research, vol. 77, pp. 508-517, April 2007.

[20] P. Kundur, Power System Stability and Control. McGraw-Hill, 1994.

[21] H. Song, B. Lee, and Y. Moon, "Reactive optimal power flow incorporating margin enhancement constraints with non linear interior point method," IEE Proceedings on Generation Transmission and Distribution, vol. 152, pp. 961-968, November 2005.

[22] T. Fortescue, L. Kershenbaum, and B. Ydstie, "Implementation of self-tuning regulators with variable forgetting factors," Automatica, vol. 17, no. 6, pp. 831835, 1981. 